Relations industrielles

Industrial Relations

\title{
Confessionnalité syndicale et régime juridique du travail dans le Québec
}

\section{Confessional Unionism and Quebec Labour Law}

\section{Gérard Dion}

Volume 15, numéro 2, avril 1960

URI : https://id.erudit.org/iderudit/1022028ar

DOI : https://doi.org/10.7202/1022028ar

Aller au sommaire du numéro

Éditeur(s)

Département des relations industrielles de l’Université Laval

ISSN

0034-379X (imprimé)

1703-8138 (numérique)

Découvrir la revue

Citer cet article

Dion, G. (1960). Confessionnalité syndicale et régime juridique du travail dans le Québec. Relations industrielles / Industrial Relations, 15(2), 162-180.

https://doi.org/10.7202/1022028ar
Résumé de l'article

Avec les développements de la législation, le syndicalisme a perdu son caractère d'institution strictement privée et volontaire. Il a acquis un caractère semi-public. Dans quelle mesure la confessionnalité syndicale peut-elle être compatible avec un tel régime juridique? Les tribunaux n'ont pas encore eu à se prononcer sur ce point. L'auteur analyse ici les différentes mesures législatives et les pratiques syndicales qui peuvent affecter la confessionnalité; il donne également une opinion doctrinale sur cette question.
Tous droits réservés @ Département des relations industrielles de l’Université Laval, 1960
Ce document est protégé par la loi sur le droit d'auteur. L’utilisation des services d'Érudit (y compris la reproduction) est assujettie à sa politique d'utilisation que vous pouvez consulter en ligne.

https://apropos.erudit.org/fr/usagers/politique-dutilisation/ 


\section{Confessionnalité syndicale et régime juridique du travail dans le Québec}

\section{Gérard Dion}

Avec les développements de la législation, le syndicalisme a perdu son caractère d'institution strictement privée et volontaire. Il a acquis un caractère semi-public. Dans quelle mesure la confessionnalité syndicale peut-elle être compatible avec un tel régime juridique? Les tribunaux n'ont pas encore eu à se prononcer sur ce point. L'auteur analyse ici les différentes mesures législatives et les pratiques syndicales qui peuvent affecter la confessionnalité; il donne également une opinion doctrinale sur cette question.

La doctrine traditionnelle de l'Eglise catholique a toujours été de recommander aux travailleurs catholiques de se grouper dans des syndicats confessionnels «sans toutefois méconnaître que des nécessités puissent obliger autrement ». (Q.A. no 38)

En notre pays, cette directive générale a été appliquée par les Evêques de la province de Québec et depuis 1921 existe une centrale syndicale catholique, la CTCC.

Le but de ce travail est d'étudier si un syndicalisme confessionnel est compatible avec le régime juridique actuellement en vigueur dans notre pays et particulièrement dans la province de Québec.

Pour en arriver là, nous allons successivement définir ce que nous entendons par confessionnalité syndicale, passer en revue les différentes lois qui peuvent s'appliquer au problème soulevé et tirer les conclusions qui nous apparaissent en découler.

LA CONFESSIONNALITÉ

Il est inutile pour les fins de la présente étude d'entrer dans tous les détails et nuances que peut comporter la confes162
DION, GÉrard, L.Th., L.Ph., M.Sc. Soc., directeur du département des relations industrielles et professeur à la Faculté des sciences sociales de l’Université Laval, Québec. 
sionnalité appliquée à un syndicat.

Le nom que porte un organisme syndical et même la présence d'un conseiller moral (quand celui-ci n'a ni droit de vote ni de veto) n'affecte en rien son caractère confessionnel. Ce qui importe, c'est da confessionnalité réelle laquelle peut se réduire à deux types.

\section{a) Groupe restreint à une confession religieuse}

Le premier type consiste dans un article de la constitution qui empêche les travailleurs qui ne professeraient pas la doctrine d'une confession religieuse (ou appartiendraient à une autre) de devenir membres du syndicat ou ne leur permet pas de bénéficier du statut de membres réguliers avec tous les droits qui y sont attachés.

\section{b) Référence à une confession religieuse}

Le second type, moins rigoureux, consiste seulement dans l'adhésion à la idoctrine sociale d'une confession religieuse grâce à un article de la constitution. Parfois on qualifie ce type de confessionnalité dinspiration.

Nous laissons donc de côté la confessionnalité de fait - i.e. lorsqu'elle n'est pas exprimée, - car au point de vue légal elle ne peut être couverte par un texte de loi.

Il est à remarquer que le problème de la confessionnalité syndicale en regard de notre régime juridique ne concerne, en pratique, que les groupements catholiques. En effet, en dehors d'une autre centrale syndicale qui réunit très peu de membres en Ontario et qui n'a aucune importance (Christian Labour Association of Canada) et de la Provincial Association of Protestant Teachers of Quebec, la CTCC, la Corporation généralle des instituteurs et institutrices catholiques de la province de Québec, l'Association des infirmières catholiques et les Syndicats forestiers de l'UCC sont les seuls groupes à posséder un caractère confessionnel selon les types indiqués.

\section{Le régime juridique}

Les lois qui sont susceptibles d'affecter la confessionnalité syndicale se ramènent à deux catégories: l'une touchant la liberté de religion et l'autre, la législation du travail proprement dite. 
a) Deux lois touchant à la Liberté de religion

\section{$1 —$ Loi sur la liberté de culte}

Chaque fois qu'il est question de religion au point de vue juridique, on doit se reporter à une pièce législative fondamentale qui garantit à chacun, en notre pays, l'exercice le plus complet de la liberté de conscience, de culte et de religion.

Depuis 1851, une telle loi est dans nos statuts. Il n'en n'a pas toujours été ainsi. Sous le régime français, la religion catholique jouissait d'une situation privilégiée. Au début du régime anglais, c'est l'Eglise anglicane qui était la religion officielle, les autres n'étant que tolérées. Me Antonio Perrault, dans une conférence prononcée en 1945 à la Semaine sociale de Montréal, a fait toute l'histoire de la liberté religieuse en notre pays. ${ }^{1}$ Il a montré particulièrement que cette liberté aocordée aujourd'hui à tous les citoyens est le résultat d'une conquête graduelle due à l'initiative des catholiques et à leur action constante menée de concert avec les confessions protestantes autres que l'anglicanisme.

Historiquement, cette liberté est venue après l'obtention des libertés civiles et politiques.

La loi fut passée par le Parlement du Haut et Bas Canada, aujourd'hui l'Ontario et le Québec, le 30 août 1851 et proclamée officiellement, après sanction par la Reine, dans la Gazette du Canada, le 9 juin 1852 .

Le préambule de la loi (14-15 Victoria, ch. 175) en indique les motifs dans ces termes:

Attendu que l'admission de l'égalité, aux yeux de la loi de toutes les dénominations religieuses est un principe reconnu de la législation coloniale; et attendu que dans l'état et la condition de cette province, à laquelle il est particulièrement applicable, il est à désirer que ce principe reçoive la sanction directe de l'assemblée législative, qui reconnait et déclare qu'il est le principe fondamental de notre politique civile...

L'article 2 se lit comme suit:

(1) Religion, culture et liberté au Canada, dans «Liberté et Libertés》, Semaine sociale du Canada, Montréal, 1945. 
Il est par le présent déclaré et statué par l'autorité susdite, que le libre exercice et la jouissance de la profession et du culte religieux, sans distinction ni préférence, mais de manière à ne pas servir d'excuse à des actes d'une licence outrée, ni de justification de pratiques incompatibles avec la paix et la sûreté de la province, sont permis par la constitution et les lois de cette province à tous les sujets de Sa Majesté en icelle. »

Il découle donc de cette loi:

a) que aucune religion ne peut prétendre à un titre officiel ou à des privilèges particuliers;

b) que chaque citoyen a le droit d'appartenir à la religion qu'il veut et de former les groupements religieux qu'il désire;

c) que personne ne peut légalement entraver l'exencice de la liberté de religion de qui que ce soit;

d) que pensonne ne peut forcer un autre à adhérer à une religion qu'il réprouve ou à en épouser les principes.

Cette loi est généralle. Comme l'indique le préambule, elle est le «principe fondamental de notre politique civile». Passée avant la Confédération, elle est sûrement encore en vigueur dans le Québec et l'Ontario, parce qu'elle n'y a jamais été abrogée et probablement dans tout le Canada. En ce qui concerne particulièrement la province de Québec, elle se retrouve dans les Statuts refondus de Québec 1941 au chapitre 307.

\section{2_La loi canadienne sur les justes méthodes d’emploi}

Depuis 1953, il existe une loi spéciale destinée à empêcher toute discrimination dans les relations du travail, que ce soit à l'embauchage, au maintien de l'emploi, aux promotions et même par l'appartenance à un syndicat.

Cette loi s'applique aux employeurs et aux syndicats ouvriers, de même qu'aux agences de placement.

Les principaux articles de la loi sont les suivants:

4. (1) Nul patron ne doit refuser d'employer ou de continuer à employer une personne, ni autrement établir contre elle des distinctions 
en matière d'emploi ou de conditions de travail, à cause de la race, de l'origine nationale, de la couleur au de la religion de cette personne.

(3) Nul syndicat ouvrier ne doit exclure une personne du plein statut de membre, ni expulser ou suspendre l'un quelconque de ses membres ou autrement établir des distinctions contre tel membre, non plus qu'en établir contre toute personne en ce qui concerne son emploi par un patron, à cause de la race, de l'origine nationale, de la couleur ou de la religion de cette personne.

(4) Nul patron ou syndicat ouvrier ne doit congédier ou expulser une personne, ni autrement établir des distinctions contre une personne, parce qu'elle a formulé une plainte ou rendu témoignage, ou prêté son concours, de quelque manière, en ce qui regarde l'introduction ou la poursuite d'une plainte ou autre procédure prévue par la présente loi.

La loi, cependant, comporte quelques exceptions. En effet, dans la définition de «patron», on exclut:

«toute oeuvre uniquement consacrée à la charité, ou organisation ou corporation uniquement philanthropique, éducative, fraternelle, religieuse ou sociale, qui ne fonctionne pas sous le régime du profit privé, ou toute organisation dirigée principalement pour favoriser le bien d'un groupe religieux ou ethnique, et ne fonctionnant pas sous le régime du profit privé. »

C'est compréhensible, car le travail exercé est de telle nature qu'il peut exiger l'appartenance à une religion particulière. Par exemple, il est normal que dans une école catholique, l'on puisse exiger comme condition d'embauchage que les professeurs soient catholiques. De même pour les hôpitaux. Ici, un cas intéressant qui ne concerne pas les catholiques se présente. C'est celui des rabbins qui abattent des volailles pour les Juifs. L'employeur, pour ce genre de travail a droit d'exiger des gens appartenant à la religion juive et satisfaisant aux exigences de cette confession religieuse. Par voie de conséquence, il est légal d'organiser des syndicats confessionnels pour de telles catégories de travailleurs.

La «Loi canadienne sur les justes méthodes d'emploi » s'applique à tout emploi portant sur des ouvrages, entreprises ou affaires qui relèvent de l'autorité législative du Parlement du Canada. Il est vrai que le nombre de travailleurs affectés n'est pas si considérable puisque dans notre pays, pour une grande part, la législation du travail relève de la juridiction provinciale. Cela n'empêche pas cette mesure d'avoir une grande importance. En effet, elle répond à la déclaration 
universelle sur les droits de l'homme aux Nations-Unies le 10 décembre 1948. L'article II se lit comme suit:

«Chacun peut se prévaloir de tous les droits et de toutes les libertés proclamées dans la présente déclaration, sans distinction aucune, notamment de race, de couleur, de sexe, de langue, de religion, d'opinion politique ou de toute autre opinion, d'origine nationale ou sociale, de fortune, de naissance ou de toute autre situation.»

Déjà d'ailleurs quelques provinces canadiennes possèdent dans leurs statuts une mesure analogue: Saskatchewan (1947), Ontario (1951), Manitoba (1953), Nouvelle-Ecosse (1956). Et il est à prévoir que dans une période d'année qui ne sera pas trop longue, toutes les provinces du pays suivront leur trace. Jusquà présent la province de Québec ne l'a pas fait. Lors de la passation de la loi fédérale, dans un éditorial intitulé «Une loi pour tous les Canadiens», L'Action Catholique de Québec donnant son approbation à cette mesure, disait: «La lutte contre la discrimination basée sur la race, la religion et la couleur est loin d'être terminée au Canada. Il faut que la loi proposée ne reste pas lettre morte et que les provinces du Canada imitent l'exemple du Fédéral et de l'Ontario, en adoptant une législation similaire applicable aux compagnies détenant des chartes provinciales».

\section{b) LA LÉgislation du travaIL}

Avant d'étudier les lois qui sont à la base de notre régime juridique des relations du travail, il est bon de remarquer que, dans notre province, exception faite pour les fonctionnaires provinciaux, nous n'avons pas de lois qui imposent des restrictions à la formation des syndicats de salariés ni à leur action de négocier avec les employeurs des conventions collectives de travail.

Des syndicats peuvent donc être créés sans autorisation préalable et se donner les statuts et règlements qu'ils désirent. Nous possédons bien la Loi des syndicats professionnels (S.R.Q., oh. 162), mais aucun syndicat n'est obligé de s'incorporer. Il peut se contenter d'être un groupement de fait sans existence juridique.

Le législateur, en général, ne s'immisce pas dans les affaires internes des syndicats et la jurisprudence tient pour valides les déterminations prises par ces institutions et les actes posés en conformité avec elles. A plusieurs reprises, des jugements sont venus confirmer cette 
prétention. Nous ne connaissons pas de cas où quelqu'un ait réclamé le droit d'appartenir à un syndicat, mais il y en a plusieurs où des travailleurs expulsés ont réclamé le droit d'être réinstallés, et, à chaque fois, les tribunaux ont jugé à partir des règlements et des constitutions existantes. $^{2}$

Mais, est-ce que cela veut dire que l'on puisse insérer n'importe quoi dans les statuts et règlements, que l'on puisse violer les libertés civiles des personnes, que l'on puisse restreindre indûment l'admission des membres?

La Loi des relations ouvrières et le Code national du Travail sont les deux pièces maîtresses qui fondent notre régime juridique des relations du travail. Comme les mêmes droits sont consacrés dans ces deux mesures, il n'est pas nécessaire de distinguer entre la juridiction fédérale et provinciale. La loi des relations ouvrières du Québec date de 1944 (S.R.Q., ch 162 a) et le Code national du travail (11-12 Geo. VI ch. 54 ; 1948) édicté en 1948 remplaçait l'arrêté-conseil 1003 qui avait été passé lui aussi en 1944 .

En 1944, la Loi des relations ouvrières est venue modifier substantiellement notre régime syndical. Parce qu'elle ne faisait que consacrer une série de pratiques courantes dans les relations patronales-ouvrières il en est bien peu qui s'en sont rendu compte au moment où cette pièce de législation d'inspiration américaine a été mise en vigueur. Mais le fait est là. Le caractère du syndicat ouvrier qui se prévaut de cette loi n'est plus le même.

Antérieurement à la Loi des relations ouvrières, le syndicat représentait uniquement ses membres auprès d'un employeur qui était libre de le reconnaître et de transiger avec lui. En pratique, cependant, lorsqu'un employeur signait une convention collective de travail, les conditions étaient étendues à tous les travailleurs de l'établissement qui entraient dans les catégories pour lesquelles le syndicat avait négocié, qu'ils soient synidiqués ou non.

(2) Encore faut-il que ces gestes ne soient pas arbitraires, même s'ils sont permis par les statuts. Dans le cas Bimson v. Johnston (1957), le président avait arbitrairement expulsé un membre en conformité avec la constitution qui lui permettait de « régler » et de «définir 》 la suspension de n'importe quel membre. Les Cours ontariennes ont donné gain de cause au plaignant en soutenant qu'un langage plus précis est nécessaire pour conférer des pouvoirs aussi drastiques impliquant des conséquences aussi drastiques. (1957) DLR. 11: (1958) 12 DLR. 379. CCH, p. 1152. 
Aujourdhui, la situation est complètement changée. Un employeur n'a plus la liberté de refuser de reconnaître un syndicat. C'est la Commission des relations ouvrières qui, lorsque certaines conditions ont été remplies par le syndicat — dont la principale est d'être majoritaire, détermine sa présentativité pour tous les travailleurs d'une unité de négociation. Notre régime légal établit le monopole de représentation pour un syndicat dans une unité de négociation.

Voici les articles de la Loi qui nous intéressent:

art. 3 Tout employeur et tout salarié ont droit d'être membre d'une association et de participer à ses activités légitimes.

art. 4 Tout employeur est tenu de reconnaître comme représentant collectif des salariés à son emploi les représentants d'une association groupant la majorité absolue desdits salariés et de négocier, de bonne foi, avec eux une convention collective de travail.

art. 5 Selon les décisions de la Commission, l'obligation prévue à l'article précédent incombe à l'employeur envers la totalité des salariés que la Commission déclare devoir former un groupe distinct pour les fins de la présente loi.

Cette consécration juridique d'un état de fait est très importante, car, légalement, les travailleurs non-syndiqués de cette unité de négociation représentée par le syndicat certifié sont obligés de se plier aux conditions de travail et à la procédure du règlement des griefs établis par le synidicat avec l'employeur. Ce syndicat n'est plus une institution privée, purement volontaire. Il aoquiert le caractère semi-public.

Les travailleurs peuvent, s'ils le veulent, rester membres d'un autre syndicat, mais celui-ci est complètement inefficace quant à ce qui a trait à la protection et à la défense de leurs droits vis-à-vis de l'employeur.

Dans notre Loi des relations ouvrières de la province de Québec, contrairement à ce qui existe dans le Code national du Travail ou dans la législation des autres provinces, il y a pourtant l'article 17 qui prévoit ce qui suit:

art. 17 Toute association réunissant au moins vingt salariés, correspondant à au moins dix pour cent du groupe soumis à une convention collective conclue par une autre association, peut soumettre par écrit au nom de ses membres, à l'employeur qui est partie de cette convention, tout grief résultant d'une violation de la présente loi ou de ladite convention: l'employeur doit immédiatement convoquer la représentant 
de l'association qui a soumis le grief, pour être entendu lors de l'examen de ce grief.

Or l'expérience a démontré que cette disposition est impraticable. D'abord, les pourcentages requis nécessitent une unité de négociation possédant au minimum 200 travailleurs. Ensuite, le syndicat minoritaire qui remplirait ces conditions n'a pas voix au chapitre lors des négociations. Enfin, dans la présentation des griefs, il a contre lui et l'employeur et le symdicat rival. Il ne faut pas se surprendre si, après seize années d'existence de la Loi des relations ouvrières, le cas est très rare où un syndicat minoritaire se soit prévalu de cet article. A toutes fins pratiques, l'article 17 est resté lettre morte.

Il est aussi nécessaire de signaler qu'aucun syndicat ouvrier n'est obligé de se prévaloir de la Loi des relations ouvrières pour s'organiser et négocier des conventions collectives. Il peut toujours ne pas s'en occuper, mais c'est une aventure risquée; l'employeur n'est pas tenu de le reconnaître et de négocier avec lui; un syndicat rival peut lui couper facilement l'herbe sous le pied et, enfin, il n'aura jamais le droit de faire une grève. Voilà pourquoi, en pratique, tout syndicat qui se veut efficace suit les dispositions de la loi.

C'est ainsi que la Loi des relations ouvrières et le Code national du Travail, qui ont pour but premier la protection de la liberté syndicale, nous amènent à nous poser les questions suivantes:

Est-ce qu'un travailleur a le droit de réclamer son admission dans le syndicat, l'unique syndicat, qui représente ses intérêts? Est-ce que ce syndicat peut poser des conditions d'admissions rendant pratiquement impossible l'adhésion des travailleurs qui sont pourtant représentés par lui?

La Loi des relations ouvrières, avec son article 3 reconnaît aux travailleurs le droit d'appartenir à un syndicat in genere et le protège contre les agissements de l'employeur. Il n'y a aucun doute là-dessus. Les termes sont suffisamment dairs.

Peut-on dire, cependant, que ce droit vaut in specie, ou, en d'autres termes, que la loi accorde aux travailleurs le droit d'être membres d'un syndicat déterminé?

Il nous semble que si nous considérons à la fois les articles 4 et 5 la question ne fait pas de doute, non plus, pour tous les travailleurs com- 
pris dans l'unité de négociation certifiée pour les représenter auprès de l'employeur. Autrement, le droit consacré par l'article 3 serait illusoire. En effet, puisque tous les travailleurs de l'unité de négociation sont représentés par un seul syndicat, lequel fixe avec l'employeur les conditions de travail (salaire, heures de travail, procédure de règlements des griefs, etc.) auxquelles chacun est obligé de se soumettre, si un travailleur veut avoir son mot à dire dans les questions qui le concernent, il ne lui reste que de faire valoir ses vues par l'entremise du syndicat certifié. Toute l'économie de notre législation du travail repose sur ce droit du travailleur d'être représenté et de pouvoir exercer un contrôle sur les affaires qui le touchent.

Le travailleur a donc le droit strict de devenir membre du seul syndicat qui représente ses intérêts auprès de l'employeur. Ceci entraîne aussi, par voie de conséquence, une certaine limitation des droits du syndicat.

Nous avons vu que la législation du travail, en général, n’intervient pas dans les affaires internes des syndicats. Mais il n'en reste pas moins que les syndicats ouvriers, comme toutes les autres institutions, doivent respecter leur nature et être soumis à ce qui regarde les dispositions générales concernant l'ondre public. Indirectement donc leurs droits peuvent être restreints.

Si la certification accordée à un syndicat par la Commission des relations ouvrières change le caractère du syndicat et que d'association privée et volontaire, celui-ci devient une association semi-publique affectant les tiers, les droits de ces tiers doivent être respectés: droit d'association, liberté de conscience, etc. Un syndicat ne peut donc pas posséder dans ses statuts et règlements des dispositions qui comporteraient, comme condition d'admission des membres et pleine jouissance des privilèges, la violation de l'un ou de l'autre de ces droits.

C'est un point de doctrine qui, à notre sens, ne fait pas de doute. Dans son Traité de droit du travail, Paul Durand, après avoir expliqué comment les «conditions d'admission sont librement déterminées par les statuts idu groupement», montre que la liberté de restreindre l'accès d'un syndicat peut être parfois limitée. Et bien que le régime syndical français soit différent 'du nôtre puisque l'on n'y a pas le monopole de représentation sur le plan de l'établissement, mais le pluralisme syndical partout, il ajoute: 
«Le principe de spécialité syndicale interdit aux groupements professionnels de faire dépendre l'admission de conditions politiques ou religieuses qui constitueraient une violation des règles relatives à l'action syndicale. Les syndicats ne sont plus toujours de simples groupements d'intérêts privés, régis par le principe de la liberté contractuelle. Ils participent à l'organisation de la profession et ont été associés à la direction de l'économie. ${ }^{3}$

Plus loin, exposant les pouvoirs du syndicat sur ses membres, il spécifie les restrictions suivantes:

« Les syndicats ne doivent violer aucune disposition d'ordre public. Ils ne pourraient contraindre leurs membres à l'accomplissement d'actes délictueux. Ils doivent particulièrement observer les dispositions impératives de la législation syndicale.

«Ils sont tenus de respecter les droits individuels de leurs membres, soit en tant qu'hommes (liberté de conscience et d'opinion), soit en tant que membres du groupe (assistance et vote aux assemblées, éligibilité aux fonctions de direction, participations aux avantages procurés par le groupement). ${ }^{4}$

Dans leur ouvrage, Droit du Travail, A. Brun et H. Galland soutiennent la même doctrine. ${ }^{5}$

L'on peut donc conclure que chaque travailleur, dans une unité de négociation pour laquelle la Commision des relation ouvrières a émis un certificat, a un droit strict à idevenir membre de ce syndicat. Les constitutions de ce syndicat ne peuvent empêcher un tel travailleur de devenir membre régulier en imposant des conditions qui violeraient leur liberté de religion.

\section{c) LeS CLAUSES DE SÉCURITÉ SYNDICALE}

Une pratique courante dans les relations du travail au Canada et aux Etats-Unis s'est généralisée avec le temps sous la pression des syndicats ouvriers. C'est celle d'insérer dans les conventions collectives de travail des clauses de sécurité syndicale.

On sait que ces clauses sont très répandues. Selon la législation fédérale, elles sont indubitablement légales. La législation québecoise est

(3) Paul Durand, Traité de droit du travail, tome III, Librairie Dalloz, Paris, 1956, pp. 214-215.

(4) Paul Dirand, op. cit., p. 221.

(5) A. Brun, H. Galland, Droit du Travail, Sirey, Paris, 1958, pp. 655-58. 
plus floue, mais, de toute façon, la plupart des conventions collectives de travail en contiennent. Un jugement récent de la Cour suprême du Canada soutient la légalité de la «Formule Rand» et ainsi, indirectement, celle de toutes les clauses de sécurité synidicale. ${ }^{B}$

Alors que selon la Loi des relations ouvrières et le Code national du travail, ainsi que nous l'avons vu, personne ne peut empêcher un travailleur d'adhérer à un syndicat certifié, ici, c'est le syndicat, qui d'accord avec l'employeur, impose aux travailleurs l'obligation d'y appartenir ou de lui apporter leur support financier, sous peine de privation d'emploi.

Si les travailleurs se voient dans une telle obligation, il est normal, en retour, que ceux-ci ne rencontrent pas d'obstacles infranchissables ou qu'on ne leur impose pas des conditions auxquelles ils peuvent selon leur conscience ou la loi refuser de se soumettre. Dans un jugement rendu par la Cour d'appel de la Colombie Britanique, en 1951, relativement à l'expulsion prétendue irrégulière d'un membre d'un syndicat qui possédait une clause de sécurité syndicale, le juge O’Halloran dit:

"A man has a right to work at his trade. If membership in a union
is a condition to working at his trade, then he has an indefeasible
right to belong to that union. It must be so, or else the union can
have no right to agitate for the closed shop. 7

Il n'est pas nécessaire que la loi positive vienne confirmer explicitement cette conclusion du droit naturel pour qu'elle soit déjà valable et respectée. Si la Loi des relations ouvrières ne spécifie rien explicitement là-dessus, de Code national du travail a cependant prévu un cas. Ainsi, en vertu ide l'article 6 (2), un syndicat ne peut expulser un travailleur ni l'empêcher de devenir membre d'un syndicat sous prétexte qu'il appartient déjà à un autre syndicat rival.

La Loi des relations ouvrières a une portée considérable. C'est elle qui marque notre régime juridique des relations du travail. Pour se protéger contre les groupements rivaux et contre les pratiques déloyales de la part de l'employeur ainsi que pour pouvoir exercer légalement le droit de grève, la plupart des syndicats se plient à ses exigences. Par ailleurs, les clauses de sécurité syndicale sont aussi un moyen couramment employé pour maintenir la force syndicale. Or l'utilisation de ces deux mesures, l'une légale et l'autre contractuelle est incompatible avec

(6) La légalité de la Formule Rand dans la province de Québec, Relations industrielles, vol. 14, no 2 (avril) 1959, pp. 280-294.

(7) Kuzyck v. White, CCH, p. 1209. 
tout ce qui peut porter atteinte à la liberté de conscience et à la liberté de religion chez les travailleurs telles qu'elles sont garanties dans la Loi sur la liberté de culte.

\section{Les références religieuses dans les constitutions syndicales}

Il faut examiner si la simple adhésion aux principes sociaux d'une confession religieuse dans les constitutions des syndicats peut être suffisante pour poser une objection de conscience à un travailleur qui n'appartient pas à cette confession religieuse.

Ceci nous mène à analyser de plus près la philosophie et l'orientation qui sont à la base des groupements ouvriers.

Il est évident, qu'on le veuille ou non, qu'il y a toujours dans tous les syndicats, énoncée ou non, une philosophie quelconque. On ne peut se passer d'avoir une conception de l'homme de la société, des rapports sociaux, du travail et même de l'économie. Même affirmer n'en pas avoir, c'est adopter une conception.

S'attendre à ce que tous les membres d'un syndicat partagent l'idéologie qui est à la base du syndicat dans les moindres détails, c'est encore impossible.

On ne peut donc pas, dans tous les cas, considérer qu'une idéologie en elle-même acceptée par un syndicat soit suffisante pour créer chez les travailleurs, matière à objection de conscience. Il faut alors distinguer entre idéologies.

Il y a certaines idéologies qui sont acceptées et partagées par l'ensemble d'une population et qui, d'une certaine manière, font partie du patrimoine culturel alors qu'il y en a d'autres qui sont formellement rejetées comme allant à l'encontre des aspirations générales parce qu'elles renversent complètement l'échelle de valeur admise dans une société.

Par ailleurs, certaines idéologies forment un corps de doctrine cohérent alors que d'autres restent floues et sans précision.

Enfin, parmi les idéologies qui forment un corps de doctrine cohérent, certaines ont, pour les interpréter et garder leur pureté, des institutions qui parce qu'elles les ont formulées ou les ont assumées, conservent sur elles un droit de regard et une autorité. A la lumière de ces distinctions, examinons quelques cas concrets. 
a) RÉFÉRENCE ì LA DÉMOCRATIE

Une référence dans les constitutions d'un syndicat à la démocratie constitue clairement une référence idéologique. Mais il ne s'agit nullement d'un corps de doctrine. Il n'y a pas non plus d'autorité pour interpréter en quoi elle consiste. Enfin, dans un pays comme le Canada, même si certains individus avaient des convictions fascistes, ils ne pourraient pas considérer cette référence comme nuisant à leur liberté de conscience.

b) RÉFÉRENCE AU COMMUNISME

Il s'agit bien ici d'un corps de doctrine qui fait un tout. De plus cette doctrine va à l'encontre des aspirations générales de la population et renverse totalement les conceptions qui font partie du patrimoine cultuturel du pays. Enfin, même s'il est possible d'imaginer un communisme sans attache à qui que ce soit, en pratique, le Parti communiste partout se considère comme l'intenprète officiel de la doctrine et de ses applications. Et dans les faits, l'expérience prouve que les individus comme les groupes qui se réclament de cette doctrine prennent leurs directives du Parti. Un syndicat communiste aocepte donc une autorité extérieure au syndicat, susceptible d'interpréter sa doctrine par dessus la tête des membres et de lui imposer une ligne de conduite dans les situations concrètes. Au strict point de vue syndical, comme au point de vue religieux, une référence au communisme suffit pour qu'il y ait matière à objection de conscience. Un tel syndicat serait donc inadmissible en regard de notre régime juridique de relation du travail. Dans les faits cependant, si certaines unités syndicales sont de tendances communistes à cause des convictions de ses dirigeants, il n'y a pas en notre pays de syndicats possédant dans leurs constitutions une référence à la doctrine communiste.

c) RÉfÉrence AU SOCIALISME

C'est encore un cas hypothétique, car il ne se présente pas en notre pays. Celui-ci est un peu compliqué, car même s'il s'agit d'un corps de doctrine, son interprétation est assez imprécise. Il y a plusieurs sortes de socialismes, les unes formellement condamnées par l'Eglise catholique, les autres n'entraînant pas de difficultés. Enfin, il n'y a pas pour le socialisme, d'autorité compétente qui est chargée d'un pouvoir effectif d'interprétation authentique en ce qui regarde la pureté de la doctri- 
ne ni de ses applications. Un syndicat qui se référerait simplement au socialisme en notre pays, à cause de cette référence dans ses constitutions, ne donnerait pas lieu à une violation des droits reconnus par notre système juridique en relations du travail.

\section{d) RÉFÉRENCE À LA MORALE SOCIALE CHRÉTIENNE}

Il en est de même pour une référence à la morale sociale chrétienne. Dans ce cas-ci, c'est un corps de doctrine dont les principes généraux sont assez précis. Elle est partie intégrante du patrimoine culturel au Canada comme aux Etats-Unis. Basée sur le droit naturel auquel se sont ajoutées les lumières de la Révélation, l'Eglise catholique s'en réclame le monopole d'interprétation authentique, mais ce droit lui est contesté par les autres confessions religieuses chrétiennes. Dans les faits, on ne peut dire qu'il y a une autorité extérieure qui s'impose.

Une référence dans les constitutions d'un syndicat à la morale sociale ohrétienne ne signifie pas l'intromission d'une autorité extérieure au syndicat. Comme il s'agit ide principes assumant le droit naturel, qui n'impliquent pas nécessairement une adhésion à une confession religieuse partioulière pas plus que l'acceptation du Christ dans sa nature divine et son rôle de Sauveur, une telle référence est acceptable par un non-chrétien et n'affecterait pas la liberté de conscience en notre pays.

\section{e) RÉfÉrence ì LA DOCTRINe SOCIALE dE L'Eglise CATHOLIQue}

Ici s'appliquent les mêmes caractères que nous venons d'expliquer au sujet de la morale sociale chrétienne. Il y a pourtant une différence fondamentale. C'est que la doctrine sociale ide l'Eglise catholique est reliée à une confession religieuse bien précise qui enseigne cette doctrine et qui a droit de regand sur elle parce qu'elle est sa propriété.

Même si, en pratique, entre la morale sociale chrétienne et la doctrine sociale de l'Eglise, il n'y a pas tant de idistance, le fait qu'il existe une autorité reconnue par tous pour l'interpréter, change complètement la nature d'une telle référence dans les constitutions d'un syndicat.

Les catholiques savent qu'en idehors de certains principes où il n'y a pas de discussion possible (principes déjà contenus dans la morale sociale chrétienne) la doctrine sociale de l'Eglise laisse place à toute une gamme d'options concrètes légitimement acceptées et même enooura- 
gées par l'Eglise. Ils savent, en outre, que l'Eglise ne se mêlera pas de questions techniques.

Mais pour les non-catholiques, il en va autrement. Ceux-ci, à tort, il est vrai, considèrent l'Eglise comme étant un tout monolithe. Parce qu'ils reconnaissent à l'Eglise le droit d'interpréter sa doctrine sociale, ils sont convaincus qu'une référence à la doctrine sociale de l'Eglise catholique dans les constitutions d'un syndicat place ce syndicat sous la tutelle d'une institution étrangère. Même s'ils n'ont aucune objection au contenu de la doctrine sociale de l'Eglise catholique, ils ne peuvent pas admettre pour le syndicat, groupement autonome dirigé de l'intérieur par ses membres, une telle interférence. Pour eux, ce serait reconnaître dans leurs affaires propres l'autorité d'une autre confession religieuse qu'ils peuvent respecter mais à laquelle ils ne peuvent en aucune manière accepter d'être soumis.

Même s'il arrive que certains non-catholiques restent indifférents à ce riroblème, objectivement celui-ci ne change pas de nature. Voilà pourquoi cette référence explicite à la doctrine sociale de l'Eglise catholique est susceptible de mettre en cause leur liberté de conscience.

Dans notre pays, tenant compte des principes de la morale et de notre législation, on ne peut donc pas forcer un non-catholique à entrer dans un syndicat possédant une référence à la doctrine sociale de l'Eglise catholique, ou à le supporter financièrement.

Enfin il serait également illégal, à cause d'un tel obstacle, de priver un non-catholique de son droit strict d'appartenir au seul syndicat habilité à le représenter auprès de son employeur. Ce serait une violation de la liberté de conscience et du droit d'appartenir au syndicat de son choix.

Un travailleur n'a pas un droit strict de travailler pour tel employeur déterminé. Si un employeur ne veut pas l'embaucher il doit cependant trouver d'autres raisons à son refus que celles de la religion, de la race, etc. Mais un travailleur possède un droit strict d'appartenir au syndicat de sa catégorie professionnelle qui représente officiellement ses intérêts si celui-ci est le seul qui est habilité à le faire aux yeux de la loi. Autrement cela reviendrait à lui nier son droit d'association. Personne ne peut donc légitimement mettre un obstacle à l'exercice de ce droit, en posant comme condition l'abandon d'un autre droit reconnu par la loi, celui de la liberté de conscience ou de religion. C'est ce qui 
arriverait pour un non-catholique si le syndicat auquel il a le droit d'appartenir possédait dans ses constitutions une référence à la doctrine sociale de l'Eglise catholique.

Même si la législation positive ne prévoyait pas ce cas, le droit naturel et la morale catholique imposeraient la même conclusion. Nous nous permettons de rappeler ici les remarques faites par le juge Casey de la Cour d'appel dans l'affaire Chabot v. Commision scolaire de Lamorandière.

«It is well to remember that the rights of which we have been speaking (right of inviolability of conscience) find their source in natural law - those rules of action that evoke the notion of a justice which « human authority expresses, or ought to express - but does not make; a justice which human authority may fail to express - and must pay the penalty for failing to express by the diminution, or even the forfeiture, of its power to command, and of which it has been said:-

But for natural law there would probably have been no American and no French revolution; nor would the great ideals of freedom and equality have found their way into the law-books after having found it into the hearts of men. »

On this point there can be no doubt for if these rights find their source in positive law they can be taken away. But if, as they do, they find their existence in the very nature of man, then they cannot be taken away and they must prevail should they conflict with the provision of positive law. ${ }^{8}$

Le fait que ce problème de la confessionnalité n'ait pas été soulevé devant les tribunaux, bien que ce régime ait été établi par la Loi des relations ouvrières depuis 1944, signifie seulement que les lois ne changent pas immédiatement les coutumes des gens.

D'abond, à plusieurs reprises, des commissions de conciliation ont refusé d'accorder des clauses de sécurité syndicale à un syndicat catholique pour ne pas violer la liberté de conscience des non-catholiques. (Cas Price Brothers).

Quant à réclamer le droit de idevenir membre d'un syndicat, d'une façon générale, les travailleurs préfèrent obtenir les avantages du syndicat, sans avoir à y adhérer ni à en payer les ootisations. Ceci explique que jusqu'à aujound'hui il n'y ait eu de cause-type devant les tribunaux.

(8) Cour d'appel; district de Québec, 1957, numéro 5156. 


\section{Conclusion}

Voici les conclusions qui découlent de cette étude.

La confessionnalité syndicale n'est pas intendite par la loi dans la province de Québec. Il peut donc se former des syndicats confessiomnels et ceux-ci peuvent représenter leurs membres en négociant des conventions collectives de travail. De plus, pour certaines catégories d'emploi où la nature même du travail connote l'appartenance à une religion particulière (c'est le cas des instituteurs), non seulement la légalité d'une organisation syndicale confessionnelle ne fait point de doute, mais sa possibilité d'action n'est soumise à aucune entrave provenant de ce caractère.

Cependant notre régime juridique est tel que le syndicat confessionnel est très limité dans ses possibilités d'existence et qu'il peut difficilement mener une action efficace. En effet, si l'on tient compte, d la fois, de la Loi ides libertés du culte, de la Loi canadienne sur les justes méthodes d'emploi, du Code national du travail et de la Loi des relations ouvrières, cette confessionnalité peut être contestée, en dehors des exceptions prévues, à chaque fois qu'il se rencontre un seul travailleur qui s'y objecte dans les cas suivants:

a) dans toutes les entreprises soumises à la juridiction fédérale;

b) dans les entreprises soumises à la juridiction provinciale lorsque le syndicat possède des clauses d'atelier syndical ou de support financier au syndicat et aussi à chaque fois que le syndicat désire se prévaloir des avantages que lui procure une certification sous l'empire de la Loi des relations ouvrières pour se protéger contre un syndicat rival, forcer un employeur à négocier et bénéficier du droit de grève.

Ce que nous venons ide dire concerne chaque syndicat particulier. Si nous considérons maintenant l'existence de tout un mouvement syndical confessionnel qui veut être puissant et prendre de l'expansion, nous estimons que le régime juridique actuel le place dans une situation très précaire, sinon impossible. Car il est difficile de bâtir un syndicalisme à contre-courant des usages acceptés par tous, surtout quand ces usages sont consacrés par la loi et vont dans le sens d'une tendance universelle.

Quant au syndicalisme idéologique, il n'y a rien dans notre régime juridique des relations du travail qui s'y oppose. 


\section{Confessional Unionism and Quebec Labour Law}

With the development of labour legislation, unionism has lost its character of a purely private and voluntary organization. It now has the status of a semi-public institution. Confessional unionism, under these conditions, can no longer stand the test of legality, even if the courts have never been called to rule on this question.

Among the laws which we have to consider here, the Labour Relations Act is the most important. This act gives to one union only the right to bargain for and on behalf of all workers in a bargaining unit. If one wants to become an efficient member of a union, he has to join the local which represents him and his fellow workers in the plant where he is employed. Moreover, compulsory membership is commonly observed in collective labour agreements as a condition of employment. The natural right of association is thus limited in fact to those who may, without making it a point of conscience, freely accept the constitution and by-laws of a union.

Local unions which require a worker to be a Catholic as a condition of membership are very seldom, but the sole fact that the Canadian and Catholic Confederation of Labour has a reference in its constitution to the social doctrine of the Catholic Church may bring a worker to scruple to belong to an affiliated local of this central body. The question of what may be a matter of conscience in this case is related to the fact that the interpretation of the doctrine is considered by non-catholics as vested with an outside organization, i.e. the Church. If the constitution of the union merely referred to the Christian social principles as the basic guide of its action, it would be a quite different thing, because this would involve no outside opinion and would be acceptable to anyone in a Christian country such as Canada. 